The Sperry Automatic Pilot was a very great aid to navigation, as it ensured that we were always on course, which is not always the case in a small vessel, short handed. It also enabled navigation and other duties to be carried out by the one person on watch; I considered it well worth two extra crew.

With a knowledge of coastal pilotage, and the working of A.N.T. (at both of which my wife also became fully proficient during the cruise), the voyage around the world did not present any undue navigational difficulties. Taking observations from a low deck in rough weather requires practice, and also judgment to know how much accuracy to expect from a particular sight. Perhaps the occasion when experience and judgment are most needed is when, after some days of overcast, one's position is uncertain, to know in what direction, and how far, one can move in safety before the weather clears. In other words, when lost, using one's head and not getting into a panic!

I was interested to learn of the simple method of navigation said to be used by the island trading schooners in the West Indies. Apparently no navigational instrument at all is carried except a pig and, if the vessel is lost, the pig is thrown overboard and apparently knows the way home.

\title{
The Use of A.P. 3270 for Surface Navigation
}

$$
\text { from A. J. R. Tyrrell }
$$

The full value of A.P. 3270 (Sight Reduction Tables for Air Navigation, Vol. I, Selected Stars, H.M. Stationery Office) does not yet seem to have been fully appreciated by surface navigators. As well as increasing the speed with which sights can be reduced, the tables offer all the advantages of a star globe or planisphere in planning a series of sights. The navigator can thus concentrate on the most suitable stars for cut, magnitude and azimuth. Since observations can be planned for a time at dusk when the horizon is still firm and the star just visible through the telescope (or, at dawn, when the star is still visible and the horizon firm), a more accurate sextant altitude can be obtained.

When planning the sights it is useful to write down the integral degrees of L.H.A. Aries for each four minutes of ship's time over the period of dawn or dusk. The six selected stars can then be extracted from the tables for the appropriate time. It is convenient to draw a rough sketch showing the relative bearings of the six stars, preferably on a chart compass rose (the D.R. position and the L.H.A. for this purpose can conveniently be those of the mid-time of the expected period of twilight). Care should be taken when this period extends over two groups of stars, as a star may disappear from the first group and another appear in the second; this can be used to advantage on certain occasions, giving an extra star for observation, so long as the 'disappearing' star is observed before the time of the change-over; if this star is needed after the change-over, extrapolation can be used for a few degrees of L.H.A. Aries, assuming a constant change of altitude. If a planet, or Polaris, or more stars on or near the meridian are necessary to complete the plan, Vols. II and III of A.P. 3270 can be used. 
The observation is straightforward. The approximate altitude is set on the sextant according to ship's time for each star in turn and the star observed on the known bearing. As each altitude is recorded with the chronometer time, the azimuth corresponding to this altitude can be taken from the tables so as to save time later when plotting.

It might seem that a selection of six stars, particularly in cloudy weather, is not enough. However, in cloudy weather the best procedure is to set the approximate altitude on the sextant in the hope of snapping the star through a break in the clouds. In clear weather six stars well selected for cut and magnitude should be all that the navigator normally requires.

When great accuracy is required, for example when only two stars have been obtained, with perhaps a poor angle of cut, or for specialized requirements such as in cable ships when the time factor is of less importance, the sights will best be worked up by the full-length formula. In this case the tables will still be useful for providing the azimuth by inspection and for use in planning the observation which should enable a better horizon and thus a more accurate altitude to be obtained. When the time factor is relatively more important than a possible loss of accuracy, the tables enable six stars to be worked rapidly and the mean position given by the intersection of the position lines will certainly yield a valuable and reliable position.

One of the great advantages of A.P. 3270 is that all the stars are used with a common argument, L.H.A. Aries, with no interpolation for declination. On the other hand the relatively large distances between the observed and assumed positions on some occasions constitutes a drawback, making the plotting a little cumbersome and open to inaccuracy. The most efficient use of a series of altitudes will be arrived at if the observer's methods are kept elastic and the most profitable combination used, each sight being considered on its own merits. For example, on some occasions it may be better to work three stars to a high accuracy rather than six using A.P. 3270 . In this case the accuracy of the final positions would probably be similar and their time of working the same. On the other hand where all observations are sub-standard it will usually be better to work six stars with A.P. 3270 than three stars more accurately. When doubt of the vessel's safety requires an urgent indication of position, two stars worked with A.P. 3270 or even one position line should be sufficient to remove uncertainty. Then a more accurate position can be worked for the necessary adjustment of course.

\title{
Plotting as an Aid to the Avoidance of Collisions at Sea
}

\author{
from Captain G. C. Forrest
}

\section{(S.S. Arcadia)}

I THIN that the impression which would be left by the Institute Working Party's Report (Journal, Vol. VII, p. 219) in the mind of any of the learned gentlemen of the Admiralty courts would be that a ship which did not keep a radar plot should be considered guilty of 'neglect of any precaution' under Article 29 of 